\title{
Effects of Surgery and Palliative Chemotherapy in the Treatment of Colorectal Liver Metastasis
}

\author{
Nigel Bird, Ruth Bird, Ali Majeed \\ Liver Research Group, Royal Hallamshire Hospital, Sheffield, UK \\ E-mail: n.bird@shef.ac.uk \\ Received May 24, 2011; revised July 8, 2011; accepted July 27, 2011
}

\begin{abstract}
Purpose: 1) to compare the survival of patients with colorectal liver metastases who underwent resection as the primary treatment against patients who had chemotherapy as the primary treatment; 2) to compare the survival in patients with recurrent inoperable liver metastases (after resection) treated with Oxaliplatin or Irinotecan against those patients with inoperable recurrence who were treated with 5-FU. Patients and Methods: 538 patients with colorectal liver metastases were referred to our unit between November 1997 and May 2005; 247 underwent liver resection and 291 had palliative chemotherapy. Results: only 7.8\% of non-operated patients survived for 5 years compared to 32.9\% of the liver resection group. After surgery, 191 patients developed inoperable recurrent disease. These patients were treated with chemotherapy; Ox/Ir patients had a 3 year survival of 55\% compared to $32 \%$ of those who received 5-FU. Our data shows that in patients who relapse after liver surgery, chemotherapy with Oxaliplatin or Irinotecan confers a significant survival benefit.
\end{abstract}

Keywords: Colorectal Cancer, Liver Metastasis, Surgery, Survival, Chemotherapy

\section{Introduction}

There are more than one million new cases and over half a million deaths from colorectal cancer in the world each year [1]. Over the course of their disease about half of all patients will develop liver metastases, with around 25\% of patients having metastases at the time of presentation (synchronous metastases). A further $25 \%-50 \%$ of patients will develop metastases in the liver during the course of their disease (metachronous metastases), typically during the first two years following the treatment of the primary cancer. Liver metastases are seen as a major cause of death in patients with colorectal cancer and, although survival is closely related to metastatic tumour burden, the long-term outlook for untreated patients is universally recognised as poor with little or no observed five-year survival [2-5].

Since the mid-1980's resection of colorectal liver metastases has become more widely performed and is now the therapeutic option of choice, representing the only chance of prolonged survival in those suitable for surgery. The first large studies from specialist centres on surgery for colorectal liver metastases reported resection rates of $20 \%-30 \%$ for all patients referred for surgery
[6,7] with 5-year survival in the range of $25 \%$ - $40 \%$ and an operative mortality of between $0.5 \%$ and $5.5 \%$. More recent studies have confirmed the efficacy of this approach, with advances in surgical technique, intra- and peri-operative care allowing the more widespread application of surgery. Peri-operative mortality rates are now in the range of $0.5 \%-2.0 \%$ whilst 5 -year survival data is commonly reported to be between $35 \%$ and $40 \%[8,9]$.

In contrast to the results from surgery, systemic chemotherapy alone offers little hope of long-term survival. Response rates for 5-Fluoruracil-based (5-FU) regimes remain static at around $20 \%$ and median survival rates rarely exceed 2 years [10-12]. The Advanced Colorectal Meta-Analysis project considered the efficacy of 5-FU in the treatment of patients with measurable non-resectable metastases who had no evidence of extrahepatic disease [13]. Covering 1400 patients from nine trials, the results suggested a $23 \%$ tumour response rate and an overall median survival of approximately 12 months for 5-FU + leucovorin.

There is unfortunately little published data concerning the use of, or strategy for post-operative chemotherapy for recurrent disease after surgical resection of liver metastases [14]. The aim of our study was to compare the 
effect of surgery and palliative chemotherapy with 5-FU and Irinotecan/Oxaliplatin in the treatment of colorectal liver metastases with respect to survival and recurrences. We evaluated the survival of all patients referred for surgical removal of colorectal liver metastases during the period from November 1997 to May 2005. In those patients on whom liver surgery was performed and who subsequently relapsed, we examined the survival of those receiving 5-fluoruracil based regimes and compared them with those who received the newer oxaliplatin/irinotecan based therapies (which were approved for routine use in the UK after April 2002).

\section{Patients and Methods}

538 patients with colorectal liver metastases from the North Trent Region were referred to our unit for assessment for surgery in the period from November 1997 to May 2005. Of these, 247 underwent partial-hepatectomy and 291 received either palliative chemotherapy or best supportive care. In the operated group there were 197 anatomical resections, of which 29 were 'extended' left or right hepatectomies, and 50 metastasectomies. Metastases were diagnosed intra-operatively at primary surgery in $27.1 \%$, by ultrasound scan in $32 \%$, CT in $31.7 \%$, MRI in $6.3 \%$ and by CEA levels in $2.8 \%$ of cases.

In the non-operated group, follow-up data were available in all 291; 4 died before being seen, 105 had extensive disease within the liver involving 5 or more lobes and therefore not considered amenable to downsizing pre-operative chemotherapy at that time, 126 had extra-hepatic disease, either peritoneal or pulmonary metastases diagnosed by contrast-enhanced CT scans, 27 were found to have benign disease and 29 refused surgery or were unfit for surgery.

Overall, $49.4 \%$ of referrals were from within the Sheffield Teaching Hospitals Trust, which equates to a resection rate per capita of 3.8 patients per 100,000 population, compared to the calculated conservative rate of 4 per 100,000 population [15]. The resection rate for the rest of the North Trent Region was 0.98 per 100,000.

\section{Chemotherapy Regimens}

The period of this study (between 1997 to 2005) was a period of great change in the availability of various che- motherapy agents and the regimens that were applied to them. We cannot therefore directly compare the efficacy of these regimens simply because of the heterogeneity of the data, we therefore present a "real -life" description of what chemotherapy was administered. The doses and cycles of the various chemotherapeutic regimes in our study were according to routine oncological practices and dose reduction and toxicity assessments were according to the Oncologists discretion and we have not specifically assessed these parameters for our study.

\section{Statistics}

Kaplan Meier estimates were performed using SPSS (v 16.0 for Mac). The Log rank test was used to determine statistical significances between groups, using the $\mathrm{R}$ language and environment for statistical computing. Differences were considered to be significant if $p<0.05$.

\section{Results}

\section{Survival}

1) Overall

The median survival for all patients who underwent resection for colorectal liver metastases was 34.4 months (95\% CI 25.75 - 43.0) at the census date September 2010; there were 173 deaths in this group, including 2 perioperative deaths. The cumulative proportion surviving for 5 or more years was 32.9\%.

In the non-operated group the median overall survival was 12.1 months (95\% CI 10.3 - 13.91); there were 209 deaths in this group overall, 36 within 3 months of referral. (Figure 1)

2) Liver Surgery + Chemotherapy (for inoperable recurrences)

Oxaliplatin and Irinotecan were introduced into routine use after April 2002 and prior to this, patients with recurrent colorectal cancer were treated with 5-FU based regimes. In our series, there were 191 patients who underwent liver surgery and subsequent chemotherapy for inoperable relapse. Of these, 143 had chemotherapy regimes based solely on 5-FU (Figure 2(a)). There were 46 deaths in this group with a median survival of 24.7 months (95\% CI 18.74 - 30.61). In the remaining 48 patients, who received oxaliplatin or irinotecan for inoper-

Table 1. Demographic data of patients in the study groups.

\begin{tabular}{|c|c|c|c|}
\hline Surgery Only & Palliative Chemo/Supportive Care & Recurrences treated with Oxaliplatin/Irinotecan & Recurrences Treated with 5-FU \\
\hline$n=247$ & $n=291$ & $n=48$ & $n=143$ \\
\hline 147 male & 169 male & 29 male & 85 male \\
\hline 100 female & 122 female & 19 female & 58 female \\
\hline Age 63.2 years (35 - 89) & Age 66.1 years (34 - 91) & Age 58.2 years (36.6 - 80.8) & Age 65.7 years (36.2 - 89.3) \\
\hline
\end{tabular}




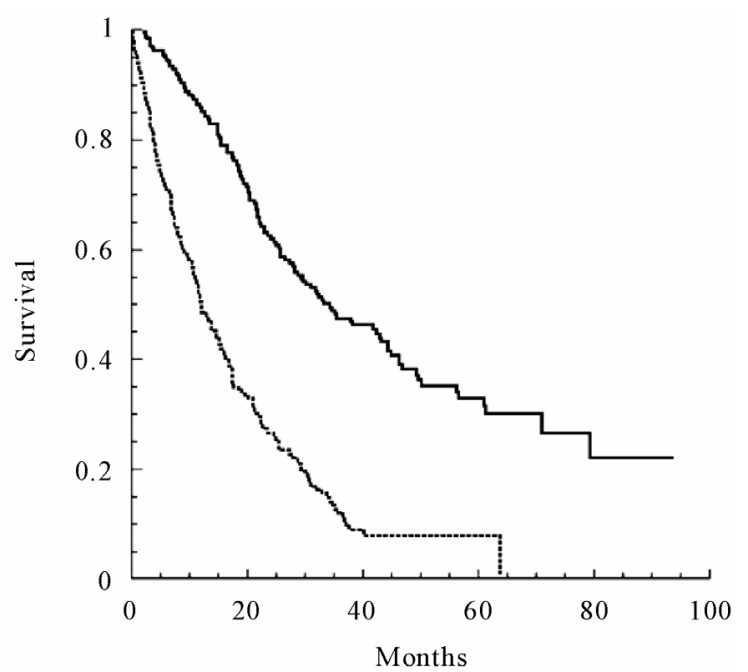

$\begin{array}{lccccc}\text { At risk } & 12 & 24 & 36 & 48 & 60 \\ \text { No surgery } & 116 & 45 & 18 & 4 & 2 \\ \text { Surgery } & 200 & 125 & 74 & 45 & 27\end{array}$

Figure 1. Kaplan Meier plots of cumulative survival proportion for 247 patients who had surgery (solid line) and 291 non-operated patients (dashed line).

able relapse, there were 23 deaths with a median survival for the group of 42.6 months (95\% CI 28.25 - 57.04).

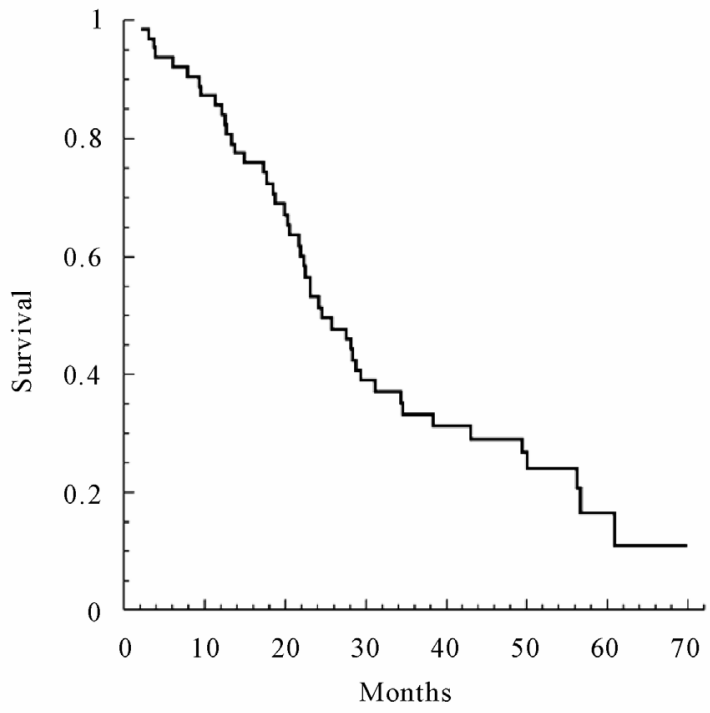

$\begin{array}{lccccc}\text { Months } & 12 & 24 & 36 & 48 & 60 \\ \begin{array}{l}\text { Surgery + 5-FU } \\ \begin{array}{l}\text { (at risk) } \\ \text { S }\end{array}\end{array} & 53 & 30 & 17 & 12 & 3\end{array}$

(a)
(Figure 2(b))

3) Chemotherapy alone

In the non-operated patients, 125 had chemotherapy with a median survival of 17.61 months (95\% CI 14.5 20.72) and 40 patients had no treatment with a median survival of 7 months (95\% CI 3.49 - 10.51). Within the chemotherapy group, there were 83 patients who had 5-FU based treatment with a median survival of 15.3 months (95\% CI 12.72 - 17.84) compared to 42 patients who had chemotherapy, including oxaliplatin or irinotecan at some stage of their disease with a median survival of 21.7 months (95\% CI 15.60 - 27.70). (Figure 3)

Statistical analysis of the survival data using the Log rank test showed the expected highly significant difference in survival between those who underwent surgery against those who did not $(p=0.0001)$.

\section{Discussion}

The major finding of our study is to show that surgery (where possible) is the most effective treatment for colorectal liver metastases. In patients that have a recurrence in the liver (and/or lungs) the survival in patients given palliative chemotherapy with Oxaliplatin and Irinotecan is significantly better than those given 5-FU. Historically, 5-FU based chemotherapeutic regimens have been the

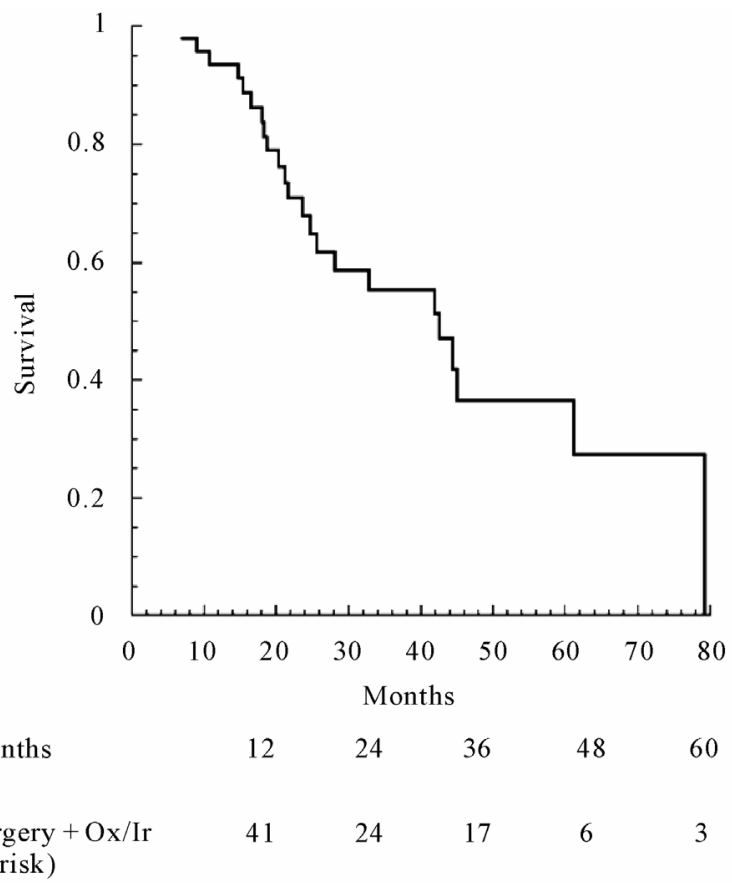

(b)

Figure 2. (a) Kaplan Meier plot of cumulative survival proportion of 62 post-hepatectomy patients who had recurrent, inoperable liver metastasis followed solely by 5FU-based chemotherapy; (b) Kaplan Meier plot of cumulative survival proportion of 48 post-hepatectomy patients who had recurrent, inoperable liver metastasis followed chemotherapy which included oxaliplatin or irinotecan. 


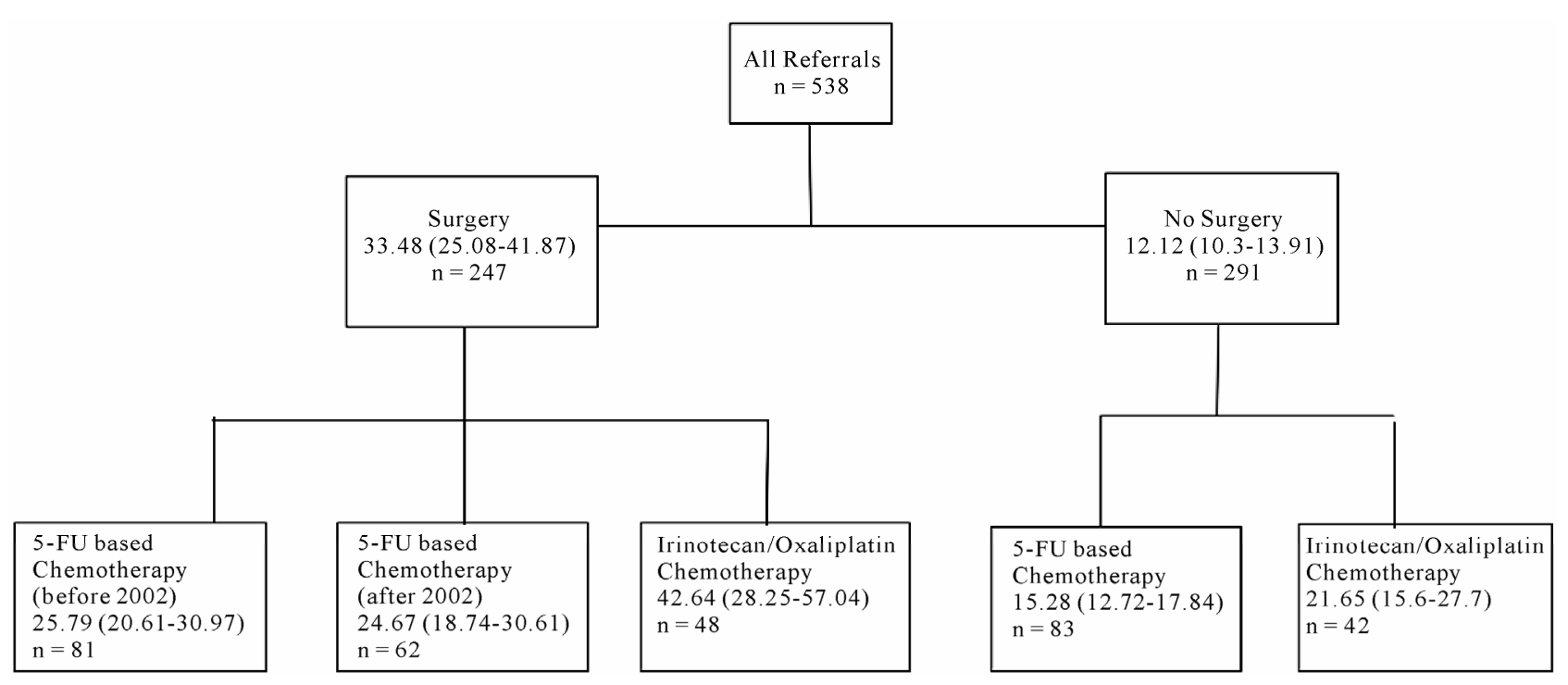

Figure 3. Dendrogram of all patients referred for surgery for colorectal liver metastasis between November 1997 and May 2005. Figures in the boxes represent median survival in months and $95 \% \mathrm{CI}$.

mainstay of treatment of recurrent colorectal cancer over many decades. Large series and meta-analyses of these treatments confirm that the effectiveness of these drugs is at best palliative, with very few complete or sustained responses obtained. The survival data of 7 months for untreated recurrent colorectal cancer and 11.7 months for 5-FU based regimens is generally accepted, with hepatic infusional chemotherapy affording a similar benefit [16]. Our results for non-operated patients given 5-FU based chemotherapy were somewhat better (median survival 15.3 months) although this could be attributed to the selection bias introduced by the fact that they were referred to the regional hepato-biliary unit for consideration of hepatic resection and thus probably had relatively small volume disease.

During the past decade or so, two major advances in the management of recurrent colorectal cancer have had a significant impact on survival and mortality from this disease. These are the introduction of surgical treatment of recurrent disease and the availability of new chemotherapeutic agents.

\subsection{Surgery}

The survival benefit from surgical resection of metastatic liver disease is clearly established with most studies showing a $25 \%$ - 39\% 5-year survival $[2,4,5,9]$. Our results of a $32.9 \%$ 5-year cumulative proportion surviving compare well with these studies. Many Japanese series show a 5-year survival of around 50\% [17,18] although European publications place the survival figure closer to $25 \%$ [19]. The benefit from lung resection for colorectal lung metastasis is less clear and although some studies show a clear survival benefit, surgical resection of these metastases is not universally accepted. The benefit from surgical treatment of loco-regional recurrence is also less clear $[18,19]$ as small numbers of patients have been treated at very few specialized centres.

\subsection{Surgery + Chemotherapy for Inoperable Recurrences}

The potential benefits of the new chemotherapeutic agents (of which oxaliplatin and irinotecan are the commonest used) in the treatment of metastatic disease has been evaluated in several randomised clinical trials but do not show a clear survival benefit with a median survival of 13 - 16 months for oxaliplatin, 15 - 17 months for irinotecan vs 11 - 14 months for 5-FU based regimens $[11,20,21]$. Whether adjuvant post-hepatectomy chemotherapy confers a survival benefit in patients who have undergone a potentially curative resection of their liver metastases is unclear. The value of adjuvant 5-FU/leu-covorin after hepatectomy has been assessed in a recently published randomised study with no difference in overall 5 year survival ( $41.1 \%$ for no adjuvant 5 -FU vs $51.1 \%$ for adjuvant 5-FU) [22]. The results from an EORTC funded study to examine the benefit of pre and post-hepatectomy chemotherapy based on oxaliplatin show a modest progression free survival advantage benefit in those receiving pre- and post-hepatectomy chemotherapy [23].

\section{Conclusions}

This study showed that surgical resection (where possi- 
ble) is the most effective treatment for colorectal liver metastases in providing long term survival. There was a survival benefit from oxaliplatin or irinotecan based chemotherapy in patients who recurred after liver resection and these recurrences were inoperable. They were therefore given palliative chemotherapy. Those patients treated with 5-FU had a significantly poorer survival than those treated with Oxaliplatin or Irinotecan. We propose that unless the value of adjuvant post-hepatectomy chemotherapy is clarified by a randomised study, patients with resectable liver and/or lung metastases should undergo surgical resection and re-resection with or without pre-operative downsizing if required. The use of oxaliplatin and irinotecan in the palliative setting should be reserved for inoperable recurrences only. This approach is likely to confer the highest chance of cure as well as a significant prolongation of survival in those that are inoperable and as a bonus, may render a small number of patients with inoperable recurrence to be subsequently operable.

We have described a consecutive series of patients with colorectal liver metastasis who underwent liver surgery (if suitable) or chemotherapy/best supportive care (if unsuitable). Our analysis suffers from the limitation that these groups of patients are probably not directly comparable because those patients who did not undergo liver surgery due to distribution of disease or fitness had, by definition, biologically aggressive disease or a weak host and their survival would therefore be expected to be worse. Patients who had liver surgery followed by inoperable recurrence and were treated with two different kinds of chemotherapy were more comparable and showed that modern drugs offer a clear survival benefit. What is not known is whether any survival benefit was conferred by surgical resection of liver metastasis for patients who later relapsed. A randomized trial comparing chemotherapy alone versus surgery alone in patients with resectable, low-volume liver involvement may show the added benefit of surgical resection, however the potential for long-term disease free survival offered by liver resection may make recruitment to such a trial difficult if not impossible. Wagner (1984) showed that patients with resectable liver disease who were not operated upon or given chemotherapy had a median survival of 21 months. Our results show modern liver surgery and modern chemotherapy for metastatic colorectal cancer can lead to a cure in up to a third of cases and significantly prolong life in the rest.

\section{References}

[1] G. D. Leonard, B. Brenner, N. E. Kemeny "Neoadjuvant Therapy for Patients with Unresectable Liver Metastases from Colorectal Carcinoma,” Journal of Clinical Oncology, Vol. 23, No. 9, pp. 2038-2048.

doi:10.1200/JCO.2005.00.349

[2] E. K. Abdalla, J.-N. Vauthey, L.M. Ellis, et al., "Recurrence and Outcomes Following Hepatic Resection, Radiofrequency Ablation and Combined Resection/Ablation for Colorectal Liver Metastases,” Annals of Surgery, Vol. 239, No. 6, 2004, pp. 818-827. doi:10.1097/01.sla.0000128305.90650.71

[3] R. Adam, V. Delvart, G. Pascal, et al., "Rescue Surgery for Unresectable Colorectal Liver Metastases Downstaged by Chemotherapy,” Annals of Surgery, Vol. 240, No. 4, 2004, pp. 644-658.

[4] G. Poston, "Surgical Strategies for Colorectal Liver Metastases,” Surgical Oncology, Vol. 13, No. 2-3, 2004, pp. 125-136. doi:10.1016/j.suronc.2004.08.001

[5] R. Adam, E. Avisar, S. Giachetti, et al., "Five-Year Survival Following Hepatic Resection after Neoadjuvant Therapy for Nonresectable Colorectal Liver Metastases," Annals of Surgical Oncology, Vol. 8, No. 4, 2001, pp. 347-353. doi:10.1007/s10434-001-0347-3

[6] J. S. Wagner, M. A. Adson, J. A. van Heerden, et al., "The Natural History of Hepatic Metastases from Colorectal Cancer. A Comparison with Resective Treatment," Annals of Surgery, Vol. 199, No. 5, 1984, pp. 502-508. doi:10.1097/00000658-198405000-00002

[7] J. Scheele, R. Stangl, A. Altenorf-Hofmann, et al., "Indicators of Prognosis after Hepatic Resection for Colorectal Secondaries,” Surgery, Vol. 110, No. 1, 1991, pp. 13-29.

[8] S. Zakharia, J. H. Donohue, F. G. Que, et al., "Hepatic Resection for Colorectal Liver Metastases," Annals of Surgery, Vol. 246, No. 2, 2007, pp. 184-191.

[9] M. A. Choti, J. V. Sitzmann, M. F. Tiburi, et al., "Trends in Long-Term Survival Following Liver Resection for Hepatic Colorectal Liver Metastases,” Annals of Surgery, Vol. 235, No. 6, 2002, pp. 759-766. doi:10.1097/00000658-200206000-00002

[10] D. Lawes, I. Taylor "Chemotherapy for Colorectal Cancer-An Overview of Current Management for Surgeons,” European Journal of Surgical Oncology, Vol. 31, No. 9, 2005, pp. 932-941. doi:10.1016/j.ejso.2005.03.015

[11] J. Y. Douillard, D. Cunningham, A. M. Roth, et al., "Irinotecan Combined with Fluorouracil Compared with Fluorouracil Alone as First-Line Treatment for Metastatic Colorectal Cancer: A Multicentre Randomised Trial," The Lancet, Vol. 355, No. 9209, 2000, pp. 1041-1047. doi:10.1016/S0140-6736(00)02034-1

[12] L. B. Saltz, J. V. Cox, C. Blanke, et al., "Irinotecan PLUS FLUORouracil and Leucovorin for Metastatic Colorectal Cancer," The New England Journal of Medicine, Vol. 343, No. 13, 2000, pp. 904-914. doi:10.1056/NEJM200009283431302

[13] Advanced Colorectal Cancer Meta-analysis Project, "Modulation of Fluorouracil by Leucovorin in Patients with Advanced Colorectal Cancer: Evidence in Terms of Response Rate,” Journal of Clinical Oncology, Vol. 10, No. 6, 1992, pp. 896-903.

[14] N. J. Petrelli, "Perioperative or Adjuvant Therapy for 
Resectable Colorectal Hepatic Metastases,” Journal of Clinical Oncology, Vol. 26, No. 30, 2008, pp. 4862-4863. doi:10.1200/JCO.2008.18.5868

[15] A. W. Majeed and C. Price, "Resource and Manpower Calculations for the Provision of Hepatobiliary Surgical Services in the UK," Annals of The Royal College of Surgeons of England, Vol. 86, No. 2, 2004, pp. 91-95. doi:10.1308/003588404322827455

[16] Colorectal Cancer Collaborative Group, "Palliative chemotherapy for Advanced Colorectal Cancer: Systematic Review and Meta-analysis,” British Medical Journal, Vol. 321, No. 7260, 2000, pp. 531-535. doi:10.1136/bmj.321.7260.531

[17] W.-S. Lee, S. H. Yun, H.-K. Chun, et al., "Pulmonary Resection for Metastases from Colorectal Cancer: Prognostic Factors and Survival," International Journal of Colorectal Disease, Vol. 22, No. 6, 2006, pp. 699-704.

[18] T. Sakamoto, N. Tsubota, K. Iwanaga, et al., "Pulmonary Resection for Metastases from Colorectal Cancer," Chest, Vol. 119, No. 4, 2001, pp. 1069-1072. doi:10.1378/chest.119.4.1069

[19] P. Girard, M. Ducreux, P. Baldeyrou, et al., "Surgery for Lung Metastases from Colorectal Cancer: Analysis of Prognostic Factors," Journal of Clinical Oncology, Vol. 14, No. 7, 1996, pp. 2047-2053.
[20] G. A. P. Hospers, M. Scaapveld, J. W. R. Nortier, et al., "Randomised Phase III Study of Biweekly 24-h Infusion of High Dose 5FU with Folinic Acid and Oxaliplatin versus Monthly Plus 5-FU/Folinic Acid in First-Line Treatment of Advanced Colorectal Cancer," Annals of Oncology, Vol. 17, No. 3, 2006, pp. 443-449. doi:10.1093/annonc/mdj104

[21] P. Rougier, E. Van Custem, E. Bajetta, et al., "Randomised Trial of Irinotecan versus Fluorouracil by Continuous Infusion after Fluorouracil Failure in Patients with Metastatic Colorectal Cancer,” The Lancet, Vol. 352, No. 9138, 1998, pp. 1407-1412. doi:10.1016/S0140-6736(98)03085-2

[22] G. Portier, D. Elias, O. Bouche, et al., "Multicentre Randomized Trial of Adjuvant Fluorouracil and Folinic Acid Compared with Surgery Alone after Resection of Colorectal Liver Metastases: FFCD ACHBTH AURC 9002 Trial,” Journal of Clinical Oncology, Vol. 24, No. 31, 2006, pp. 4976-4982. doi:10.1200/JCO.2006.06.8353

[23] B. Nordlinger, H. Sorbye, B. Glimelius, et al., "Perioperative Chemotherapy with FOLFOX4 and Surgery versus Surgery Alone for Resectable Liver Metastases from Colorectal Cancer (EORTC Intergroup Trial 40983): A Randomized Controlled Trial,” The Lancet, Vol. 371, No. 9617, 2008, pp. 1007-1016. doi:10.1016/S0140-6736(08)60455-9 